\title{
Implementation of Android Control Bluetooth Spy Car with LIVE Video Streaming
}

\author{
Md. Harun-Ar-Rashid \\ Department of Computer \\ Science and Engineering \\ Mawlana Bhashani \\ Science and Technology \\ University \\ Santost, Tangail, \\ Bangladesh
}

\author{
Md. Wahidur \\ Rahman \\ Department of Computer \\ Science and Engineering \\ Mawlana Bhashani \\ Science and Technology \\ University \\ Santost, Tangail, \\ Bangladesh
}

\author{
Rahabul Islam \\ Department of Computer \\ Science and Engineering \\ Mawlana Bhashani \\ Science and Technology \\ University \\ Santost, Tangail, \\ Bangladesh
}

\author{
Md. Tarequl Islam \\ Lecturer, Department of \\ Computer Science and \\ Engineering \\ Khwaja Yunus Ali \\ University, Enayetpur, \\ Sirajganj, Bangladesh
}

\begin{abstract}
Android application provides some features to establish communication with peripheral devices. We developed android smartphone control Spy Car with live video streaming \& Storing. Our developed car contains following features (i) Controlling Car via android application in four direction (ii) Live Video Streaming (iii) Rotating Camera $-180^{\circ}$ to $180^{\circ}$ (iv) front LED control. Our developed Car controls via Bluetooth using USART protocol real time video streaming by Wi-Fi. It can be workable in search mission purpose.
\end{abstract}

\section{Keywords}

Arduino UNO, Smart Phone with Android Operating System, Bluetooth Module, Wireless Camera (IP Camera Nano), Motor Driver, Motor.

\section{INTRODUCTION}

Now a days android mobiles are very popular in the present world. The most significant part of this mobile is the Operating system. Android Operating Rules through the world with his greatest features. As we know that, Android is a mobile operating system which is developed by Google, basically based on the Linux kernel and initially started for touchscreen mobile devices such as smartphones and tablets. Moreover, we know that android operating allows user's enriched with some extra features rather than other operating system such as touch screen, finger print security, controlled mobile by gesture, eye movement security etc. In android mobiles, there's an ordinary feature named Bluetooth to operate the phone wirelessly as well as data exchanging process. The most effective part of this mobile for operating wirelessly, with high speed data transmission rate which is called Wi-Fi. Data transmission rate through this process is very comfortable, efficient and smooth.

In this scenario, we designed a Car that can operate wirelessly by Android mobile. This Research paper has represented how a Car controlled through Bluetooth and live video streaming which is operated through Wi-Fi by an Android application.

Many papers focus on mainly Controlling Car through Bluetooth. But they did not provide any video streaming. [1] Some other papers focus on controlling car by Microcontroller only. The authors in paper [2] design a wireless car using an application without providing any concept of live video streaming. In paper [3], strongly focuses in IP Camera and Wi-Fi. In paper [4], the authors tried to find out modern technology of Bluetooth communication.

\section{MOTIVATION OF WORK}

The main intention of this research to raise awareness on Robotics. We tried to develop a Spy car that can perform and can be used for our daily life. We dreamed, a simple Car that is controlled wirelessly by android application for our various purposes. This simple architecture may be constructed by a student, even general people without any trouble to perform in investigation. This low-cost project can rapidly use in security system by Law and Enforcing Agencies.

\section{DESCRIPTION OF REQIREMENTS 3.1 Arduino UNO}

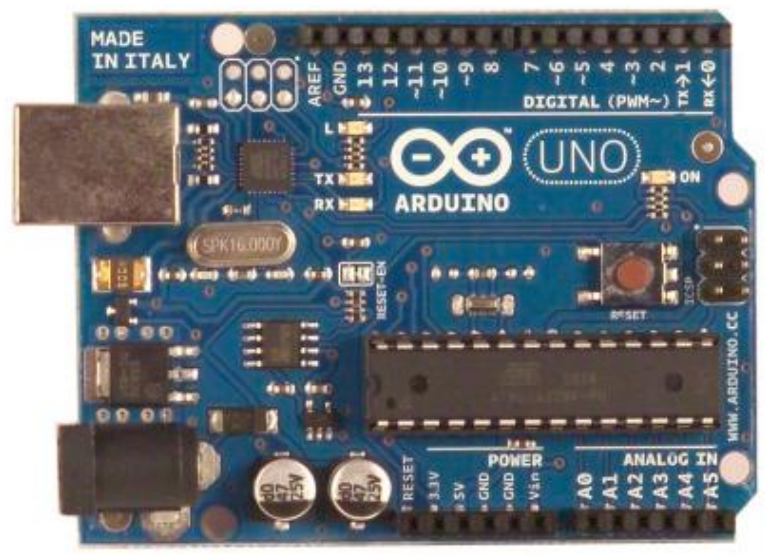

Figure 1: An Arduino Uno Board

The Arduino Uno use a microcontroller named after ATmega328.As we know that it contains 14 digital input/output pins, there's a set of 6 individual pins known as analog, a crystal oscillator which has frequency of $16 \mathrm{MHz}$, a DC power jack, an ICSP header, a USB connection, and a reset button. It has two output pins known as RX and TX. It has also GND (ground pins) and VCC (distributed voltage). In our research, we use 6 digitals pins where 2 to 5 pins respectively used for motor controlling and 13 pin used for controlling camera by servo motor and last 12 pin used for controlling head light. 


\subsection{Motor Driver L298N}

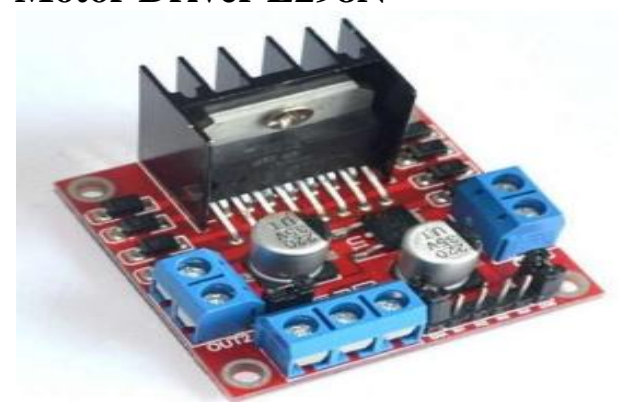

Figure 2: Motor Diver L298N

This Motor driver allows DC motors speed as well as direction. This L298N is used to distribute the voltage between two DC Motor. This L298N allows voltage form 5V up to $35 \mathrm{DC}$. There's a transistor that can convert voltage on board from up to $12 \mathrm{~V}$ to $5 \mathrm{~V}$. It has 2 output points. It has 1 VCC (5V to $35 \mathrm{~V} \mathrm{DC),} \mathrm{GND} \mathrm{(ground} \mathrm{pin),} \mathrm{and} \mathrm{regular} \mathrm{5V} \mathrm{pin.}$ It has two outputs which can operate two motors. L298N has 4 input pins to operate the DC motors.

\subsection{Bluetooth HC-05}

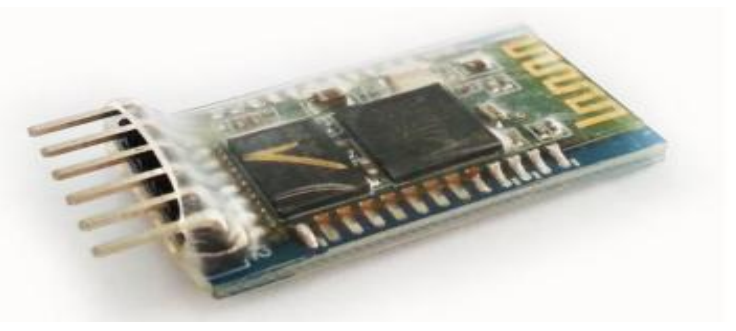

It's known to all; Bluetooth communication is the most vulnerable wireless communication process for short range. [4-5] Bluetooth module HC-05 is very simple to use as Bluetooth Serial Port Protocol (BSPP) which ensures transparent wireless connection and communication system. It contains 4 pins named as VCC, GND, TX (Connect with the MCUs), RX and a KEY. We connect TX to arduino RX and $\mathrm{RX}$ to arduino $\mathrm{TX}$, provide $5 \mathrm{~V}$ dc supply from arduino as well as GND.

\subsection{Motors}

The Electric motors took a revolutionary change in last few decades. Now a days, almost every mechanical movement needs an electrical motor to drive our everyday life. Electrical motors are not only used for mechanical movement but also produce and store mechanical energy. Now in various purposes, hundreds of devices are accomplished in our regular life by an electric motor. We use motor in automobiles, robot, hand power tools, fan, washings machine, food blender even in medical science. In this way, we use two DC motor in our Spy Car.

\subsection{Rechargeable Battery}

Rechargeable Li Po Battery is very effective in the field of Robotics and power management system. It can store energy and produce DC voltage supply. We use 2 cell 3.7 Volt battery that gives us almost $7.4 \mathrm{~V}$ and $2400 \mathrm{~mA}$.

\section{FLOW CHART \& BLOCK DIAGRAM}

This figure: 1 shows about the architecture flow chart of our research and also the block diagram.

Fig 3: Bluetooth HC-05 Module

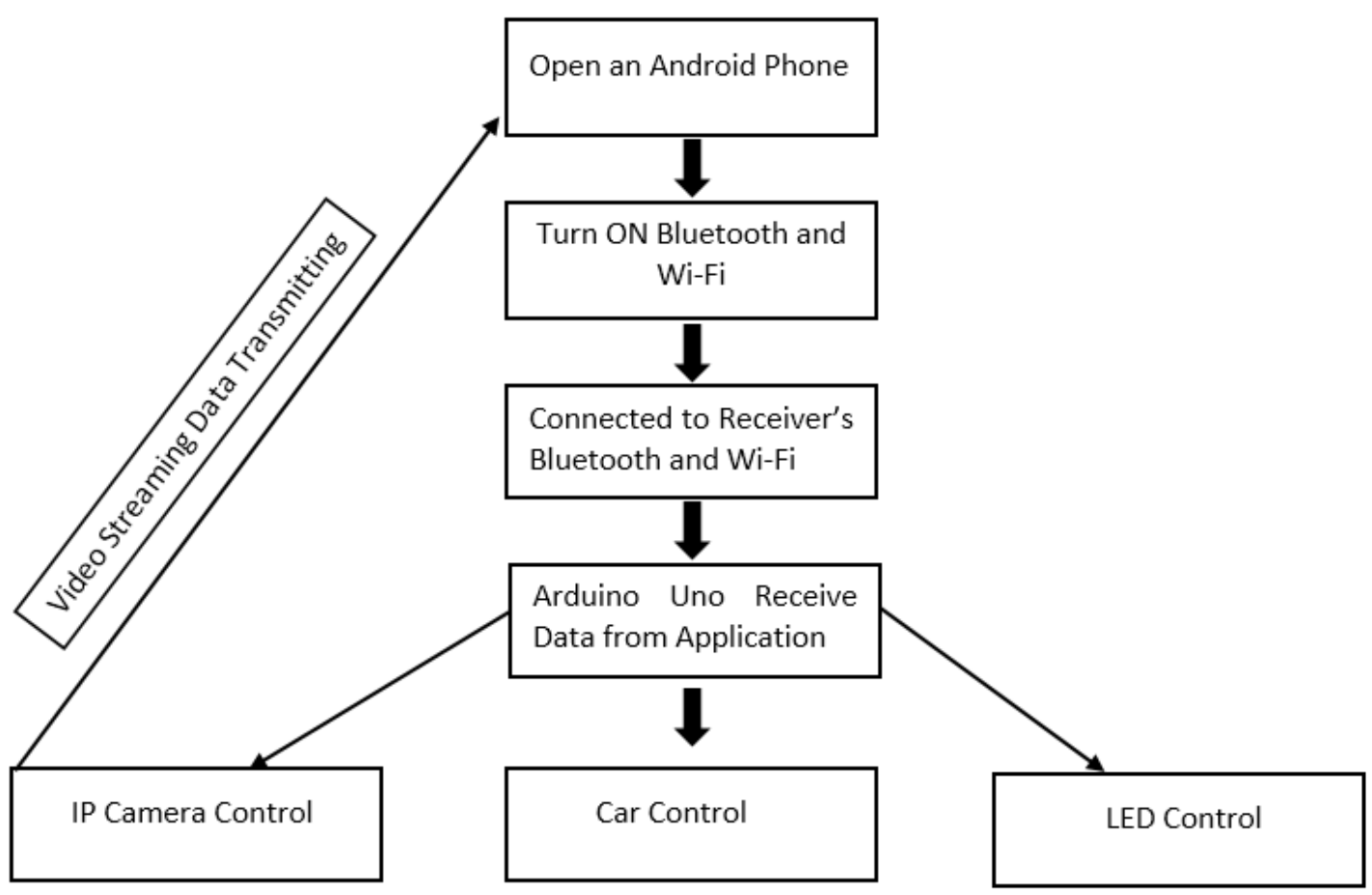

Figure 4: Flow Chart of Spy Car 


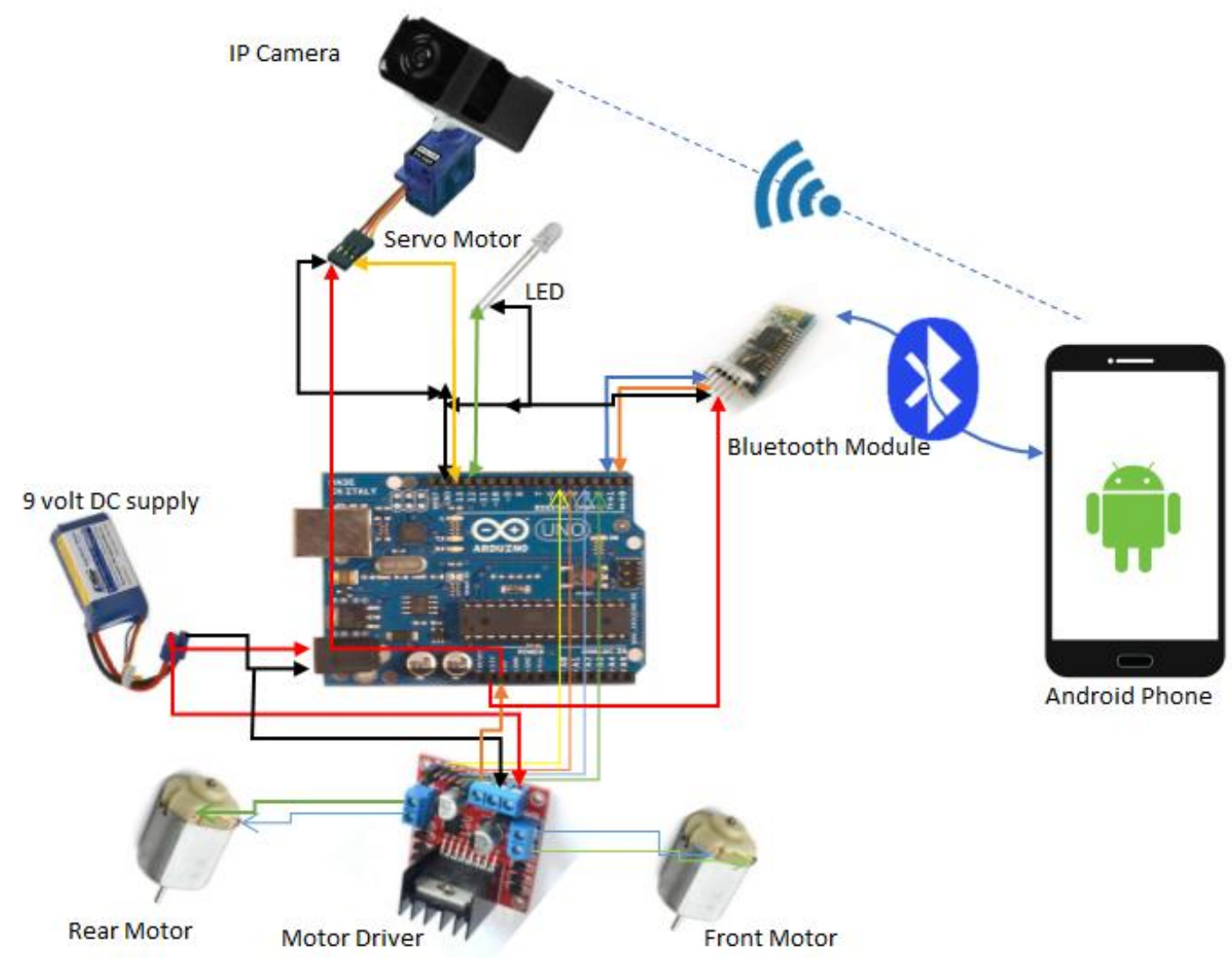

Figure 5: System Architecture of Spy Car

\section{WORKING PRINCIPLES}

5.1 Firstly, one should open the app ensured that Wi-Fi and Bluetooth connection are established.

5.2 Secondly, Switch on the Car, make sure that if the device is paired with the android mobile. We can customize the password of HC-05 is " 1234 ".

5.3 Thirdly, press on the "Start Spy Car", there are 3 part of the apps

\subsubsection{Video streaming monitor:}

Wireless video streaming process can be performed by Wi-Fi with IP camera. Where android application communicates camera through Wi-Fi. [3] Video streaming performed from server to client side.

\subsubsection{Car control option}

5.3.3. Camera Control

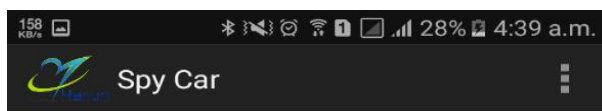

\section{Spyicar}

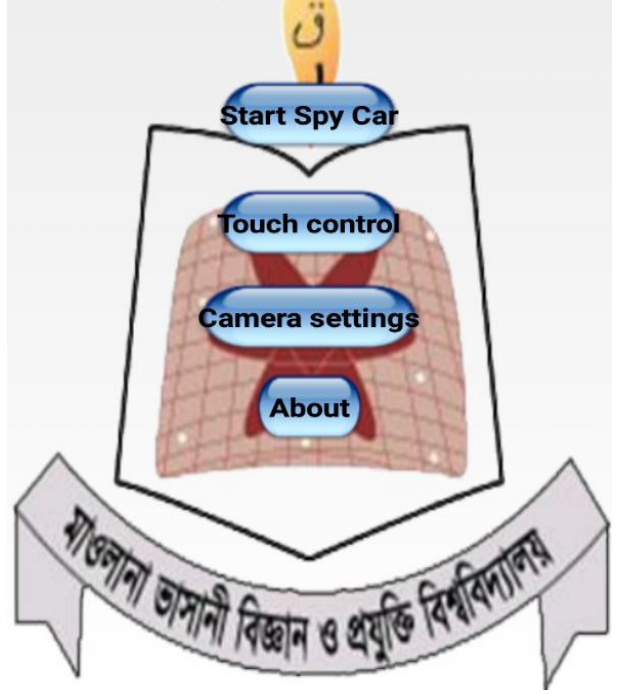

Figure 6: Home Page of our developed Application At Car control option:

5.4 Fourthly, at car control option, while pressing the "Up" button, it transmit a character type data ' $F$ ' through Bluetooth. After receiving the character ' $F$ ', arduino commands the car to move "Forward". 
5.5 While pressing the "Down" button, it transmit a character type data ' $\mathrm{B}$ ' through Bluetooth. After receiving the character 'B', arduino commands the car to move "Backward".

5.6 While pressing the "Right" button, it transmit a character type data ' $\mathrm{R}$ ' through Bluetooth. After receiving the character ' $R$ ', arduino commands the car to move "Right".

5.7 While pressing the "Left" button, it transmit a character type data ' $\mathrm{L}$ ' through Bluetooth. After receiving the character 'L', arduino commands the car to move "Left".

5.8 While pressing the "Break" button, it transmit a character type data ' $\mathrm{B}$ ' through Bluetooth. After receiving the character 'B', arduino commands the car to stop".
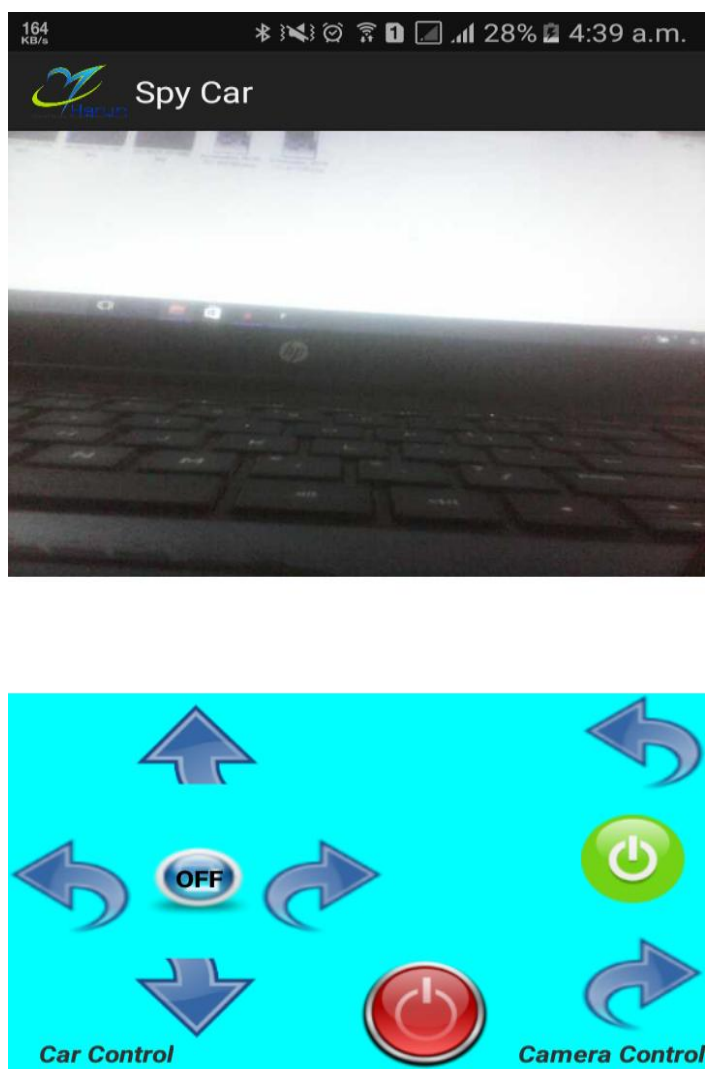

Figure 7: Car Control Option with Live Video Streaming

At Camera Control:

5.9 While pressing the "Right" button, it transmit a character type data ' $A$ ' through Bluetooth. After receiving the character 'A', arduino commands the servo to move camera towards "Right".

5.10 While pressing the "Stable" button, it transmit a character type data ' $\mathrm{H}$ ' through Bluetooth. After receiving the character ' $\mathrm{H}$ ', arduino commands the servo to move camera "Neutral".

5.11 While pressing the "Left" button, it transmit a character type data ' $C$ ' through Bluetooth. After receiving the character ' $\mathrm{C}$ ', arduino commands the servo to move camera towards "Left".

5.12 Finally, while pressing the "Middle" button, it transmit a character type data ' $D$ ' through Bluetooth. After receiving the character 'A', arduino commands the light to set ON. Again, pressing the same button it transmit ' $\mathrm{E}$ ', arduino commands the light set OFF.

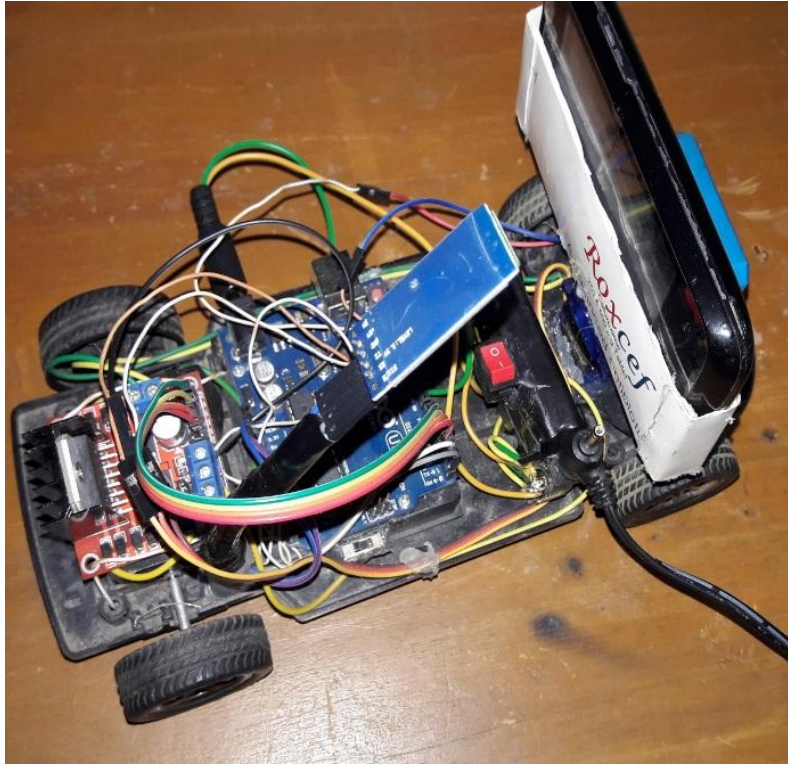

Figure 7: Prototype of Spy Car

\section{APPLICATION LEVEL}

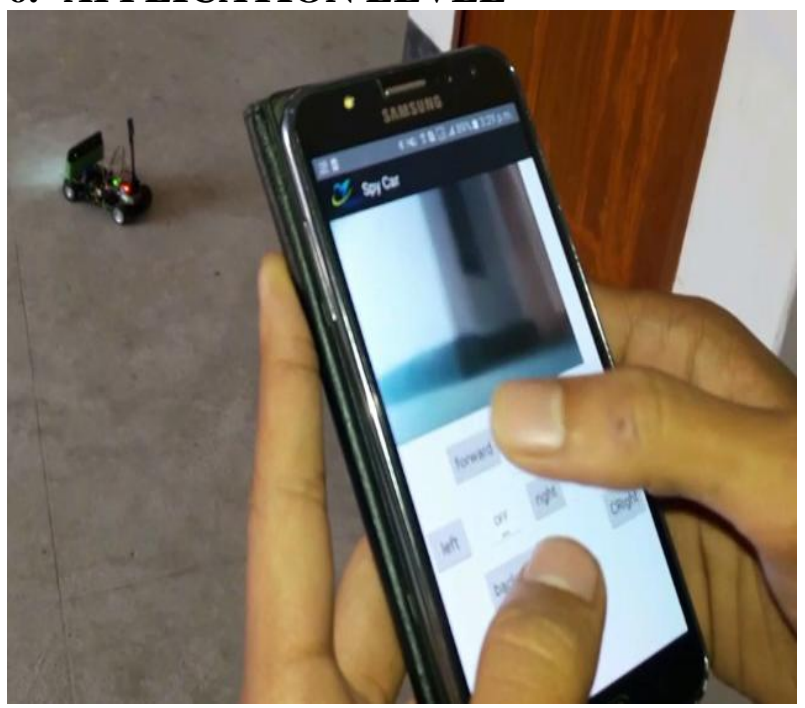

Figure 8: An User drive Spy car using application

At present this kind of project is worthwhile to mankind in various aspect of their life. The project's main significance is to ensure security and investigation. People can watch live streaming effortlessly even it is feasible to record the video along with audio this types of tiny robot we can operate even in narrow pipelines, drain and water tank. The car can be used effortlessly by Army or Law Enforcement Agencies They can engage and put through in their investigation matter. The main fact of this car is the low-cost rate, so general people also can afford this and apply it to their activities. Young people also can have it for driving it through android application and can feel the taste of digital world.

\section{FUTURE WORK}

We wish to dream up this car far more than that you can imagine. We use Wi-Fi hotspot to transmit data live streaming. If we use router, then it will be possible to watch the live streaming through some others phone of computers. It's also possible to build a website for video streaming for security purpose. By adding GPS tracker, we can also easily 
find the location of the car. This features might be added our application for future purposes.

\section{CONCLUSION}

We worked hard and it was very challenging to build a Spy car that can operate by users wirelessly via android application that can transmit data as real time video streaming with audio. This research allows rewarding with great experience, arise huge confident in our mind, meet with brief ocean of knowledge. We believe that this prototype might help the people in social and economic security purpose and reconnoiter needs.

\section{REFERENCES}

[1] Gandotra, S., Sharma, B., Mahajan, S., Motup, T., Choudhary, T. and Thakur, P., 2016. Bluetooth
Controlled RC Car using Arduino. Imperial Journal of Interdisciplinary Research, 2(9).

[2] Pahuja, R. and Kumar, N., 2014. Android Mobile Phone Controlled Bluetooth Robot Using 8051 Microcontroller. International Journal of Scientific Engineering and Research, 2(7), pp.14-17

[3] Ramesh, S., 2015. Reliable Video Streaming over Wi-Fi (Part 1).

[4] Singh, P., Sharma, D. and Agrawal, S., 2011. A Modern Study of Bluetooth Wireless Technology. Dept. of Computer sci. \& Eng. Raipur, (Chhattisgarh).

[5] Sairam, K.V.S.S.S.S., Gunasekaran, N. and Redd, S.R., 2002. Bluetooth in wireless communication. IEEE Communications Magazine, 40(6), pp.90-96. 